\title{
DEFENSA NACIONAL
}

\section{Gral. E.P.(r) Edgardo Mercado Jarrín}

\section{Visión critica de su contenido}

Los comentarios siguientes en su primera parte, visión crítica, se refieren a los conceptos de Seguridad Nacional y Objetivos Nacionales cuya competencia y responsabilidad de formulación no han sido abordados por la Constitución, y al análisis de las atribuciones del Presidente de la República y la diferenciación de las misiones que cumplen la Fuerza Armada y Fuerzas Policiales.

La Constitución de 1979 no define lo que se entiende por Seguridad Nacional y sólo trata de ella dos veces: en el Título III al ocuparse del Régimen Económico, Art. 114, que expresa, "por causa de interés social o seguridad nacional, la ley puede reservar para el Estado actividades productivas o de servicio"; y luego con propiedad y pertinencia, en el Cap. XII de la Defensa Nacional y el Orden Interno, Art. 269, que precisa "El Estado garantiza la Seguridad de la Nación mediante la Defensa Nacional".

Como hay que garantizar la obtención de los Objetivos Nacionales eliminado o neutralizando las presiones dominantes, internas y externas, que se le oponen, aparece la Seguridad.

El surgimiento de nuevas formas de agresión, la necesidad de resolver de acuerdo a criterios de justicia los problemas sociales y económicos, la influencia de ideologías foráneas y la necesidad de un mínimo de libertad de acción, dan nuevas dimensiones a la protección de la nación. De ello dimana la distinción entre la idea de Seguridad y el concepto clásico de Defensa. La primera es una condición, un estado, que inclusive condiciona la ejecución de la segunda. La Defensa es una acción específica y definida; un acto directamente ligado a determinado tipo de amenaza. La finalidad de la Seguridad Nacional es proporcionar a la Nación un grado de garant ía para alcanzar el desarrollo. Por Defensa Nacional debe entenderse un género de medidas que el Estado adopta para lograr la Seguridad Nacional, un medio para alcanzarla; Seguridad que a su vez es un valor intermedio para la consecución y mantenimiento de los Objetivos Nacionales. Seguridad no es pues, el apresto militar aunque lo incluye; Seguridad no es la actividad militar tradicional, aunque la comprenda. La noción de Seguridad, es más amplia que la de Defensa. La Seguridad es responsabilidad de todos; la Fuerza Armada es un elemento esencial de la Defensa Nacional. El concepto de Seguridad Nacional podría expresarse, como:

La garantía que el Estado otorga a la Nación mediante acciones políticas, económicas, sociales y militares para la obtención y mantenimiento de los Objetivos Nacionales, a pesar de las presiones dominantes existentes.

La Seguridad Nacional garantiza la defensa de nuestros intereses 
económicos, la dignidad nacional, la oposición a ideolog as ajenas a nuestra realidad y la facultad de actuar con independencia tanto en el campo intemo como externo. Cuando se consigue satisfacer, en la medida de las posibilidades, los intereses y aspiraciones de la Nación y los Objetivos Nacionales se hallan a cubierto de interferencias sustanciales, particularmente las presiones dominantes existentes, tanto internas como externas, la Nación disfrutará de una adecuada seguridad.

Los conceptos de desarrollo y Seguridad están permanente y estrechamente unidos. Sin desarrollo no hay Seguridad y viceversa. La falta de desarrollo atenta contra la seguridad y la falta de ésta atenta contra las posibilidades de alcanzar aquél. El C AEM expresa "estas políticas son concomitantes y se diferencian en sus fines, objetivos, acciones y medios". Pero una cosa es cierta: la Seguridad requiere un proceso contínuo de desarrollo $\mathrm{y}$ en todo caso al estadista corresponde alcanzar los Objerivos Nacionales sin detrimento de una ni de otra.

La Constitución no sólo ha omitido en su texto este fin fundamental del Estado, sino que tampoco lo ha considerado entre los deberes del Estado ni de la persona. El Estado cumple dos fines concomitantes e interdependientes en el desarrollo de su política general: el bienestar general, finalidad suprema y la seguridad integral, finalidad consecuente. En el Título II, el Estado y La Nación, Cap. I., Art. 80, en donde se definen sus deberes primordiales se trata del bienestar general pero no se precisa el de la Seguridad Nacional, aunque se hace referencia a la defensa de la soberanía nacional. Igualmente en el Título I, Derechos y deberes fundamentales de la persona, Cap. VIII de los deberes, Art. 76 , se expresa que "todos contribuyen al bienestar general", pero se omite expresar su aporte a la Seguridad Nacional. Esta es una omisión tanto más inexplicable cuanto precisamente la tendencia mundial y continental de las modernas Constituciones es referirse a la Seguridad Nacional y sus organismos superiores responsables reciben también este nombre. Estimamos que debió dársele más profundidad al Capítulo XII que con propiedad debió denominarse: Seguridad Nacional, Fuerza Armada y Fuerzas Policiales y establecer claramente en él que:

"La Seguridad Nacional es de la competencia y responsabilidad del Estado; y que toda persona natural o jurídica, son igualmente responsables de la Seguridad Nacional, dentro de los límites señalados por la ley.

La Constitución al no haber hecho referencia a qué se entiende por Seguridad Nacional, deja un gran vacio difícil de llenar pues no se sabe cuál debe ser su naturaleza, ni cuáles de sus alcances serán asegurados por la Defensa Nacional. Una de las finalidades de la Seguridad Nacional es garantizar el desarrollo y como consecuencia de ello se infiere que la Defensa Nacional debe a su vez garantizarlo, pero, ¿cualquier tipo de desarrollo? De la lectura del Preámbulo de la Constitución hay que concluir que se trata de garantizar un desarrollo independiente y con justicia social. 
En dicho preámbulo se precisa que estamos "Decididos a promover una sociedad justa, libre y culta, sin explotados ni explotadores...", y luego en párrafo aparte se continúa "Decididos, asimismo..., a la cancelación del subdesarrollo y la injusticia;... "De manera que inter-relacionando lo que expresa el Preámbulo y lo que señala el Artículo 269 podemos concluir que:

el Estado garantiza la seguridad de la Nación mediante la Defensa Nacional y como la Seguridad a su vez es garantia del desarrollo, la Defensa Nacional debe garantizar un desarrollo no dependiente y con justicia social, de acuerdo a la Constitución de 1979.

La Constitución de 1979 señala que el ejercicio de la Defensa Nacional se realiza a través de un sistema cuya organización y funciones determina la ley. El funcionamiento de la Defensa Nacional requiere el establecimiento de un Sistema de Defensa Nacional a cuyos componentes hay que señalarles atribuciones y responsabilidades. Este sistema ha sido creado por D.L. No. 22653 de agosto de 1979, y en el se establecen los organismos del Estado responsables: a nivel nacional (Consejo, Secretaría y Servicio de Inteligencia nacionales); a nivel dominio (Comités interministeriales); a nivel Fuerza Armada (Comando Conjunto de la Fuerza Armada y Ministerios).

La Constitución no hace mención a los Objetivos Nacionales y políticos, ni a quién le compete la tarea de formularlos. La única norma, entre nuestra frondosa legislación, que hace referencia a ellos es la nueva Ley de Defensa Nacional, asignando al Consejo de Defensa Nacional la atribución de participar en su formulación. Nuestra Carta Política debió consignar al efecto un artículo del tenor siguiente:

"Compete al Consejo de Defensa Nacional formular los Objetivos Nacionales y los Objetivos Políticos".

Los Objetivos Nacionales constituyen la concreción de los intereses y aspiraciones vitales, que en una determinada etapa histórico-pol itica, toda la nación busca satisfacer teniendo en cuenta sus posibilidades y peculiaridades. Se refieren usualmente a aspiraciones e intereses vitales que subsisten durante largos períodos, como una meta a ser alcanzada sin plazo determinado. El bien común, es el valor máximo a ser logrado cuyo entomo, más que a la pol íca, a la filosofia compete precisar. Este objetivo sintesis, puede ser reducido a dos macro-objetivos que se integran y son interdependientes: el desarrollo para alcanzar el bienestar, actualizando valores, generando el cambio social y el progreso de la Nación; y, de otro lado, la seguridad garantizando el desarrollo, procurando la conservación, la justicia social, el equilibrio, la ley, el orden y la libertad de decisión.

Los Objetivos Nacionales sirven de orientación general a la Política Nacional. Los gobiernos realizan en cada periodo una política que necesita de objetivos más precisos, deducidos de los objetivos nacionales. La política 
gubernamental se atiene a los medios disponibles del Poder Nacional con que se cuenta para alcanzarlos y mantenerlos. Surgen asi los objetivos políticos, actuales o inmediatos, que en relación a los objetivos nacionales constituyen etapas intermedias, relacionados con ellos, condicionados por la coyuntura que vive el país, caracterizados por ser realistas y formulados de acuerdo a la capacidad del poder nacional, dada la imposibilidad de atender al cumplimiento de los objetivos nacionales.

Un sistema Nacional de Planificación requiere una coordinación muy estrecha entre el desarrollo y Seguridad. La formulación e integración de las políticas de desarrollo y Seguridad, que se realiza a nivel nacional con la participación de los Organos de Seguridad y de planificación, carece de orientación sin el apropiado establecimiento de los Objetivos Nacionales y de los objetivos políticos, y la Constitución debió precisar a quienes compete su formulación.

La nueva Constitución es más explícita que la anterior en lo que concierne al Presidente, pues inequivocamente señala que es, además, el Jefe Supremo de la Fuerza Armada y de las Fuerzas Policiales y que dirige el Sistema de Defensa Nacional. Este es un aspecto de gran trascendencia, sobre el cual queremos profundizar porque a él se le quiere dar una connotación "política" adjetiva, cuando realmente responde a profundos conceptos doctrinarios y entraña una gran responsabilidad ya que significa la perentoria necesidad de precisar los "Propósitos estratégicos" que en definitiva es tarea del Presidente de la República asesorado por el Consejo de Defensa Nacional y en general por el Sistema de Defensa Nacional.

Las normas militares deberán derivarse de la política nacional. Si se formulan por consideraciones castrenses unicamente, es posible que no sirvan a ningún fin político. La Fuerza Armada deberá prepararse, organizarse, instruirse para alcanzar fines pol íticos determinados, propósitos estratégicos definidos. Después que una Nación determina la clase de influencia que desea ejercer o las condiciones que desea establecer, sólo entonces pueden desarrollarse las armas, las fuerzas y las estrategias que sirvan a estos propósitos.

La guerra es un instrumento de la política y la continuación de ésta; por lo tanto, aquella es de responsabilidad de quién conduce ésta, vale decir del estadista y no del jefe militar. La participación de la política se expresa en la definición de los propósitos estratégicos nacionales, que se denominan objetivos políticos y finalidad de la guerra, y en la elección de los métodos y formas de hacer la guerra. Dirigir y conducir la guerra es responsabilidad de la política; son atributos del estadista, a quien incumbe preverla, definir su finalidad, guiarla y pactar los tratados de paz, de acuerdo a-los requerimientos del objetivo político cuya determinación y actualización también son su responsabilidad. Y este y no otro es el profundo significado y los alcances del Art. 273o. de la nueva Constitución. 
El comportamiento nacional depende en gran medida de si los propósitos estratégicos nacionales han sido formulados con sapiencia y habilidad en función del interés nacional. Las energías de una nación para ser convenientemente encausadas, reclaman el diseño de una estrategia general. Frente a una estrategia determinada, el apoyo de la voluntad nacional puede ser entusiasta y la opinión pública expresarse en forma unificada o ellas pueden ser dubitativas o inciertas. La situación geopolítica del Perú exige, compulsivamente, la definición de sus propósitos estratégicos nacionales para tratar de alcanzar la unidad y cohesión nacional, sin los cuales resulta dificil proceder con eficiencia y continuidad en el campo de las relaciones internacionales. El grado de firmeza en la conducta internacional y la solidaridad y cohesión en una nación, son las causas principales de su éxito o de su fracaso. Y esto es responsabilidad de la política del Presidente, y son los alcances de la nueva Constitución, a pesar de las omisiones señaladas.

En la nueva Constitución, la Fuerza Armada y las Fuerzas Policiales están equiparadas y colocadas en un plano de igualdad lo cual es correcto. Pero es incurrir en un simplismo tratar de reducir sus tareas que les compete diciendo "en el caso de las Fuerzas Armadas, su misión principal es la defensa nacional y su misión subsidiaria es asumir el control interno" $Y$ en el caso de las Fuerzas Policiales ocurre al revés: "su misión principal es el mantenimiento del orden y su misión subsidiaria participar en la Defensa Nacional". Y tal parece haber sido la mente de los constituryentes al enumerar en el mismo capítulo y en forma correlativa sus diferentes artículos referentes a estas instituciones estableciendo un paralelismo, aunque no una identidad, en sus misiones. Lo apropiado hubiera sido poner cada asunto en su lugar dividiendo el Capitulo en tres subtítulos: uno dedicado a la Defensa Nacional; otro a la Fuerza Armada; y, un tercero, a las Fuerzas Policiales.

La Fuerza Armada es, en un caso extremo, la guerra, la utilización legítima de la fuerza, de la violencia, para asegurar la supervivencia del Estado y sus instituciones. La Fuerza Armada pertenece al Estado en cuanto es uno de sus pilares básicos y garantía del ordenamiento normativo; pertenece a la nación en cuanto está integrada por ciudadanos de todas las clases sociales y de todas las regiones del pais. Se tipifica por las armas, su poder de aniquilamiento, paralización o inutilización de la Fuerza Armada del enemigo como finalidad estratégica. Ante todo importa discernir que ella es uno de los elementos del Poder Nacional, es decir es uno de los recursos que dispone la nación para alcanzar y mantener sus Objetivos Nacionales a pesar de los antagonismos y presiones existentes.

Las Fuerzas Policiales, supone, en el Estado el poder de normar la conducta pública de las personas sin el ejercicio de la violencia. Las Fuerzas Policiales pueden pertenecer al Estado, a un Estado Federal, a una región o a una localidad; no necesariamente se integran por ciudadanos de todas las regiones del país. Lo que tipifica a las Fuerzas Policiales es su capacidad de protección, de control y de orientación de la población nacional. Las Fuerzas Policiales no 
constituyen un elemento del Poder Nacional ni a ellas puede asignárseles una finalidad estratégica, ni la de asegurar los Objetivos Nacionales.

La tecnoiogia ha obligado a abandonar el antiguo concepto de que una nación necesita un pequeño núcleo de militares muy bien adiestrados capaces de entrenar, en el arte de la guerra, tanto a sus reservas como a las Fuerzas Policiales cuando un conflicto bélico es inminente. Ahora la Nación debe tener su Fuerza Armada lista y organizada de acuerdo a los Objetivos Nacionales en capacidad de hacer frente a una acción sorpresiva, lo que importa un mayor profesionalismo y una distinción más profunda en las misiones, los requerimientos de entrenamiento, equipamiento y medios, de la Fuerza Armada y de las Fuerzas Policiales. Los manuales de instrucción y los reglamentos del empleo de las nuevas armas no son fáciles de entender y la manera de manejarlas resulta mucho más compleja.

Tal como está concebido y redactado el Cap ítulo sobre Defensa Nacional, la Constitución deja expedito el derecho para reclamar la creación de los Ministerios de los diferentes Institutos Policiales, a imagen y semejanza de los actuales de la Fuerza Armada.

La Constitución omitió integrar los actuales Ministerios de la Fuerza Armada en uno solo para la mayor eficiencia de su misión, el que estaría encargado de establecer y conducir la política de Defensa Nacional y asegurar su cumplimiento en armonía con la política general del Gobiemo. De esta manera ha conservado el Perú el "privilegio" de ser uno de los pocos paises en el mundo en que existen Ministerios separados de la Fuerza Armada. Esto no significa que la Constitución cierre el paso a la formación de un Ministerio de la Defensa Nacional, ya que puede crearse mediante la ley respectiva a tenor de lo prescrito en el Art. 214. Los constituyentes pudieron disponer su funcionamiento incluyendo el precepto pertinente en forma similar a lo hecho con la Amazonía (Art. 120), cuando señala que "una institución técnica y autónoma tiene a su cargo la investigación, la evaluación y el control de los recursos".

A continuación expondremos brevemente algunas justificaciones en apoyo de su creación:

Guerra Integral. - La guerra moderna es de carácter integral. La estrategia militar es la ciencia y el arte del empleo de la Fuerza Armada en forma conjunta para alcanzar los Objetivos Nacionales mediante el empleo de la fuerza. Hay que elegir los escasos medios disponibles, combinarlos y hacerios converger coordinadamente, para que concurran a un mismo resultado. Esto exige un planteamiento y una conducción bajo una sola autoridad, que se concreta en la unidad de dirección, tanto en la paz como en la guerra. La reunión de fuerzas es factor de poder, su dispersión es signo de debilidad. Cada día, debido a los adelantos tecnológicos, las armas y sus sistemas resultan comunes para la Fuerza Armada, como en el caso de la c oheter $\mathbf{n}$. Por tal razón surge una nueva tendencia que se orienta a desaparecer las diferencias y aun la división clásica de 
la Fuerza Armada -Ejército, Marina y Aeronáutica-y reemplazarla por un solo Instituto constituido por especialidades genéricas. En Israel, pais que ha conducido en los últimos treinta años, cinco guerras victoriosas- su Fuerza Armada no sólo es dirigida por un Ministro de la Defensa, sino que está integrada por un Estado Mayor único y bajo cuya jefatura están subordinados los Comandantes de cada Instituto.

Por otro lado la mayor í de los países del mundo tienen el mando unificado de su Fuerza Armada, bajo un Ministerio de la Defensa Nacional, de donde resulta el Perú un caso aislado. Consecuentemente se atenta contra el principio de unidad de dirección, que requiere la guerra integral, si se mantiene el actual sistema de Ministerios separados de Guerra, Marina y Aeronáutica. Por lo demás este principio de la unidad de dirección no es privativo de la Seguridad Nacional ni de la Fuerza Armada. Es un requisito indispensable para la buena marcha de cualquier empresa. La ciencia de la administración así lo tiene establecido.

Racionalización del gasto.- El equipamiento de la Fuerza Armada es consecuencia del planteamiento operativo que corresponde al Comando Conjunto de la Fuerza Armada (CCFA) y su Estado Mayor. Los recursos del país son escasos y hacen difícil cumplir por lo general con dicho planeamiento. Como el Presidente del CCFA no tiene asiento en el Consejo de Ministros para justificar las adquisiciones requeridas, podría darse el caso de que no se le reconozca la prioridad que le corresponde, decidiéndose realizar otras adquisiciones, únicamente por razones de la política particular de uno de los Institutos Armados. Un Ministerio de la Defensa alejará este peligro.

En muchos países este Ministerio está a cargo de un civil, que sigue las recomendaciones del CCFA o del Jefe de Estado Mayor Conjunto, cuya jefatura se ejerce rotativamente entre las más altas autoridades de cada uno de los Institutos Armados, y con ello se eliminan las "susceptibilidades" que resultan de la distribución de los recursos para el equipamiento y que atentan contra la racionalización del gasto y la consecuente economía en la adquisición de armamentos.

Fuerzas balanceadas.- El país requiere de fuerzas debidamente balanceadas. Deficitario en alimentos, dependiente del tráfico marítimo; y por razones de carácter geohistórico, de desarrollo y seguridad, tiene que complementar su posición geoestratégica con un eficiente Poder Naval. Con difícil orografía y concentrada en Lima Metropolitana el $800 / 0$ de la economía del pa is, donde se ubican los elementos vitales cuya destrucción y desorganización provocarían el aniquilamiento de la potencialidad de la nación y con ello quebrantarian su voluntad de lucha, en la eventualidad de un conflicto, un adecuado Poder Aéreo tiene grandes incidencias, reales y efectivas en la supervivencia del país. Nuestras fronteras abiertas, de escaso valor defensivo, apreciables recursos periféricos y gran sensibilidad estratégica, reclaman un Poder Terrestre que contrarreste las vulnerabilidades que ellas ofrecen. Por todo ello, geopolítica y estratégicamente, el pais requiere de una Fuerza Armada 
debidamente balanceada, lo que obviamente es más fácil de obtener en forma económica, mediante el mando unificado. Es muy diferente el caso de la Unión Soviética que necesita la preeeminencia del poder terrestre, o los EE.UU., que requieren un potente poder naval; sin embargo, a pesar de ello, estas grandes potencias mantienen el mando unificado mediante la existencia de sendos Ministerios de Defensa.

Cabe formularse esta pregunta: ¿Es compatible con la unidad de concepción y de empleo de la Fuerza Armada que requiere la guerra moderna y la racionalización de los gastos en la adquisición de armamentos que continue la presencia de tres ministerios diferentes? Considero que la respuesta es que conviene al interés nacional el funcionamiento de un solo Ministerio de la Fuerza Armada.

\section{Comparación con la Constitución de 1933}

La Constitución de 1933 en su Art. 213o., que dice "Asegurar los derechos de la República y el cumplimiento de la Constitución", institucionalizó la suprema misión de defender igualmente la soberanía y la Constitución y dio amplias funciones a la Fuerza Armada que la podían convertir, como efectivamente ha sucedido en respuesta a imperativos circunstanciales, en el árbitro de los destinos del país.

Una vasta misión que desbordaba la naturaleza y la finalidad para la cual fue creada la Fuerza Armada - salvaguardar los más grandes intereses de la Nación- y que dio lugar en el pasado a toda clase de interpretaciones y actitudes incluyendo, la intervención política. La nueva Constitución ha eliminado este dispositivo y por ello creemos que era aún más necesario precisar el concepto de Seguridad Nacional, para su mejor conocimiento y compenetración espiritual, y no señalar únicamente que el Estado garantiza la Seguridad de la Nación mediante la Defensa Nacional.

La nueva Constitución prescribe que la Fuerza Armada y las Fuerzas Policiales no son deliberantes y agrega que todas ellas están sometidas al Poder Constitucional. Esta disposición está orientada a fortalecer la prescindencia política de las Fuerzas Militares, por considerar que deben constituir la gran reserva moral de la Nación y merecer la confianza, el respeto y el afecto del pueblo al que sirve.

Las relaciones entre la autoridad civil y la Fuerza Armada resultan por su naturaleza cada vez más complejas y en circunstancias, por excepción, como ha sucedido históricamente, pueden desbordar las estrictas especificaciones lógicas de los juristas. Hablamos de circunstancias, porque la lógica constitucional no proviene de un mero marco teórico, procede de la realidad y de la historia, y todos sabemos que por excepción, en situaciones de desorden, la Fuerza Armada ha sido, entre nosotros, un factor político y deliberante en lo atinente a los asuntos públicos, al gobierno del Estado. 
Las disposiciones reglamentarias establecen que las órdenes se cumplen sin dudas ni murmuraciones, lo que al trascender al público ha dado la imagen de rigidez en el comportamiento intelectual castrense. Sin embargo, la actividad de Estado Mayor ha introducido en la metodología de la toma de decisiones el trabajo en equipo, y el oficial expone sus planteamientos a través de estudios, apreciaciones, informes, recomendaciones, etc. resultado del análisis de los hechos y factores que hacen al problema y que reflejan sus puntos de vista, su criterio y su capacidad profesional. Una vez adoptada la decisión por el superior responsable, aunque ella sea diferente a sus recomendaciones, en virtud de la disciplina el oficial de Estado Mayor reorienta su trabajo en provecho de su cumplimiento, dejando de lado las razones que hubieren motivado su posición adversa.

La disciplina es la base en que descansa la estructuración de las Fuerzas Militares y una de sus exteriorizaciones es la subordinación que no implica solamente obediencia al superior jerárquico, sino también al Poder Constitucional que presupone el acatamiento a las autoridades constituídas, a las leyes y a la voluntad popular, principio fundamental de la República y de la cultura pol ítica.

En relación al equipamiento de las fuerzas militares se autoriza al Estado para que cualquiera sea la política gubernamental, se reserve los fondos correspondientes de manera que no se deje a la Defensa Nacional sin los recursos para satisfacer sus requerimientos. Al mismo tiempo se prescribe que no puede cambiarse el destino de estos recursos, quedando prohibida su transferencia a otros pliegos de la administración.

Otra diferencia con la Constitución de 1933 es la nueva disposición referente a la participación en el desarrollo económico y social del país y la defensa civil de acuerdo a ley. La Fuerza Armada de hoy, por el nivel tecnológico que viene alcanzando, donde los hombres ya no sólo se ordenan de acuerdo a su jerarquía, sino de acuerdo a sus funciones, se aproxima al tipo ideal de la empresa industrializada. La Constitución no ha hecho sino recoger una realidad: su participación en el desarrollo. Las tareas que en este ámbito cumple la Fuerza Armada, pueden sintetizarse en las siguientes: hace del soldado un hombre útil a la empresa; participa en el establecimiento de la infraestructura económica (carretaras, cartografía, colonización, apoyo a la explotación petrolífera, etc.); colabora a tonificar la economia local; contribuye a que el pais desarrolle sus actividades en ambiente de paz y armonía.

En cuanto a los ascensos se han introducido las modificaciones siguientes: corresponde al Senado la ratificación de los ascensos y no al Congreso como era anteriormente; la ratificación ya no incluye a Coroneles y Capitanes de navio; se ha eliminado la atribución que ten í el Congreso de conceder ascensos por servicios eminentes sin el requisito de la propuesta del Poder Ejecutivo.

Aunque en la nueva Constitución se ha limitado la intervención política en este 
campo, lo correcto hubiera sido suprimirla. En efecto, hoy día existe un sistema de ascensos que eitrana de las nuevas técnicas de administración de personal, que hace de la promoción de los oficiales tanto un requisito para llenar las vacantes producidas por las necesidades del servicio como un estímulo y recompensa al rendimiento y la capacidad profesional. Los procedimientos en la Fuerza Armada que hacen del mérito el pilar básico del ascenso, han demandado que idénticos procedimientos se apliquen en el país como lo prueba el hecho que tanto el Servicio Diplomático, el Poder Judicial, como el Magisterio, han reclamado para sí y puesto en práctica disposiciones similares a las que hoy rigen a la F.A. La ratificación por el Senado desvirtúa el carácter eminentemente técnico de los procedimientos de ascensos y con ello se mantiene la posibilidad de que por motivaciones distintas a la capacidad e idoneidad profesional se concedan o nieguen los ascensos, como ha sucedido en anteriores oportunidades.

En lo que se refiere al Fuero Militar, la Constitución ha recogido el reclamo de la opinión pública y se ha dispuesto que los Fueros y el Código de Justicia Militar se apliquen sólo a los miembros de la Fuerza Armada y Policiales por delitos referidos a su función y a los civiles unicamente en caso de guerra exterior.

La Constitución en su Art. 670., al igual que la de 1933, declara en suspenso el ejercicio del sufragio para los miembros de la Fuerza Armada en actividad. El argumento que más frecuentemente se esgrime en su contra es que "significaría una peligrosa politización de la F.A". Tal argumento es contradictorio con la realidad histórica, pues precisamente la F.A. es la institución que ha tenido la mayor intervención en la conducción política del país. Creemos que la participación en las elecciones le dar áa al Oficial una razón más para respetar el veredicto popular y fortalecer su sentido de civismo. El ejercicio del poder se delega mediante el sufragio en el personal gubernamental. El oficial está muy identificado con el poder; se le inculca que es la habilidad para prevalecer en el conflicto, superar los obstáculos y obtener los efectos deseados, por eso cuando al militar no se le permite expresar sus preferencias políticas a través del voto, se le está negando la posibilidad de contribuir a personificar en quién o quiénes tendrán en sus manos el ejercicio del poder político supremo: la soberan ia, de la que se siente su guardián permanente.

El cada vez mayor profesionalismo de la F.A. evitaría que el ejercicio del sufragio favorezca la politización de la F.A. y coadyuvará a mantenerla al margen de la política. A medida que la tecnología aumenta en complejidad y la estrategia reclama mayor flexibilidad, se acrecientan las exigencias de mejor educación y adiestramiento para el personal de las Fuerzas Militares. Los nuevos niveles profesionales y el servicio militar selectivo crean ahora mejores condiciones, en relación con su participación electoral, para cerrar el paso al partidarismo político en los cuarteles y al autoritarismo que pudiese constreñi el voto. 


\section{Reflexión final}

Entre 1968 y 1974 se dieron pasos históricos irreversibles. Nadie puede desconocer que la revolución cambió al Perú. En el marco de una crisis económica mundial y ante la transferencia del poder a la civilidad, gruesos errores se cargan al pasivo del gobiemo militar. Sin embargo, los resultados de éste son materia más apta para la justicia y tranquilidad de la reflexión histórica, que sabrá reconocer sus aportes y diferencias sus fases reales y los errores que hubo, pero lo que cuenta es el saldó positivo.

La Fuerza Armada reconoce que, al volver a sus cuarteles, su prestigio está cuestionado pues no se pudo alcanzar equilibradamente los objetivos de la revolución. Pero fue necesario que pusiera todo su empeño y vocación de servicio para tratar de elevar el nivel de vida del pueblo, conseguir un mayor bienestar y una posición internacional digna e independiente,

En todo caso, conviene que los líderes de esta hora piensen seriamente en la naturaleza y carácter de la Fuerza Armada peruana, tras doce años globalmente transformadores. Porque bien podría ser que la paralización del desarrollo de su "profesionalismo participatorio" se convierta en una peligrosa frustración. Al nivel actual de complejidad del Estado y de la Ad̉ministración Pública y dada la incrementada intemacionalización de cualquier sistema político, es riesgoso aferrarse al concepto de Fuerza Armada con "funciones tradicionales", de carácter simplemente instrumental.

Si se acepta que los profesionales castrenses van a seguir manteniendo una posición analítica frente a la vida peruana, es inevitable aceptar que continuarán alertas ante la consecución de los objetivos nacionales. Por tanto, no es posible pensar en un retorno simplista al "profesionalismo apolítico" de antaño y la nueva Constitución debió definir algún tipo de participación racionalizada que evite, precisamente, periódicos desbordes o interferencias militares, en la acción de los gobiernos civiles, ya que media en unos y otros la misma preocupación por la seguridad y el bienestar colectivo. 